\title{
Myopic and perfect foresight in the OLG model
}

\author{
Philippe Michel* $\quad$ David de la Croix ${ }^{\dagger}$
}

May 17, 1999

\begin{abstract}
We compare the dynamics under myopic foresight and under perfect foresight in an overlapping generations model with capital accumulation and two-period lived individuals. When the rational dynamics are defined and the myopic dynamics are monotonic, the study of myopic foresight allows to characterize the dynamics under perfect foresight. The study of myopic dynamics also allows to obtain a condition of uniqueness of the steady state that bears only on the second order derivatives of the utility and production functions.
\end{abstract}

JEL Classification numbers: E32, O41.

Keywords: perfect foresight, myopic foresight, stability.

*IUF, Université de la Méditerranée and GREQAM, Centre de la Vieille Charité, rue de la Charité 2, F-13002 Marseille, France.

${ }^{\dagger}$ National Fund for Scientific Research and IRES, Université catholique de Louvain, Place Montesquieu 3, B-1348 Louvain-la-Neuve, Belgium. E-mail: delacroix@ires.ucl.ac.be. The financial support of the PAI programme $\mathrm{P} 4 / 01$ is gratefully acknowledged. We thank Raouf Boucekkine for his comments on an earlier draft. 


\section{Introduction}

In a standard overlapping generations model with capital accumulation (Diamond, 1965) there is in general a multiplicity of stationary equilibria (Galor and Ryder, 1989). In the simple case of a logarithmic utility function, the capital stock of the next period is proportional to the wage of young agents. As the wage function is not necessarily a concave function of existing capital (for instance with a CES production function with low substitution possibilities) multiple stationary states can arise.

With a logarithmic utility function the dynamics are the same for any type of expectation formation. With a more general utility function however, the savings function is defined over wages and the expected interest rate. The positive stationary states are the same for both perfect and myopic foresight but the dynamics are different. In this paper we address the issue of the relationship between the dynamics under myopic foresight and those under perfect foresight. We show that this comparison is interesting as knowledge of the dynamics under myopic foresight allows, under certain conditions, to know the dynamics under perfect foresight.

\section{Perfect and myopic foresight}

In a standard overlapping generations model with capital accumulation, we denote the life-cycle utility function $u(c, d)$ where $c$ is consumption when young and $d$ consumption when old. The savings function $s(w, R)$ is derived from the maximization of the life-cycle utility subject to the inter-temporal budget constraint and depends on wages and the interest rate. It is defined as the implicit function

$$
u_{c}^{\prime}(w-s, R s)=R u_{d}^{\prime}(w-s, R s)
$$

The perfect foresight equilibrium is defined as follows:

Given an initial capital stock $k_{0}$, an inter-temporal equilibrium with perfect foresight is a sequence of capital $\left(k_{t}\right)_{t \geqslant 0}$ that satisfies for all $t \geqslant 0$

$$
\begin{aligned}
R_{t+1}^{e} & =R_{t+1}=f^{\prime}\left(k_{t+1}\right) \\
k_{t+1} & =\frac{1}{1+n} s\left(\omega\left(k_{t}\right), R_{t+1}\right)
\end{aligned}
$$

where $k$ denotes per capita capital. $R_{t+1}^{e}$ denotes the expectations on the factor of interest $R_{t+1}, f($.$) is the production function in intensive form, n$ is the growth rate of the population and $\omega\left(k_{t}\right)=f\left(k_{t}\right)-k_{t} f^{\prime}\left(k_{t}\right)$ is the wage function. Hence, at the inter-temporal equilibrium with perfect foresight the stock of capital of period $t+1$ should verify the following implicit equation:

$$
(1+n) k_{t+1}-s\left(\omega\left(k_{t}\right), f^{\prime}\left(k_{t+1}\right)\right)=0 .
$$

The existence of such equilibrium can be proved under fairly general assumptions on the technology and preferences. For the equilibrium to be unique the following additional

assumption should be made: 


\section{Assumption H.}

For all $w>0$ and all $k>0$,

$$
\Delta(k, w)=0 \Longrightarrow \Delta_{k}^{\prime}(k, w)>0,
$$

where

$$
\begin{aligned}
\Delta(k, w) & =(1+n) k-s\left(w, f^{\prime}(k)\right) \\
\Delta_{k}^{\prime}(k, w) & =1+n-s_{R}^{\prime}\left(w, f^{\prime}(k)\right) f^{\prime \prime}(k)
\end{aligned}
$$

One important difficulty for applications is that the hypothesis $\mathbf{H}$ is not directly formulated in terms of preferences and technologies but depends on the equilibrium values of $k$ and $w$. A more appealing assumption on preferences is when the utility function verifies $\forall c>0, u^{\prime}(c)+c u^{\prime \prime}(c) \geqslant 0$. This condition is equivalent to assuming that the inter-temporal elasticity of substitution $\sigma(c)$ is greater or equal to 1 . This implies that $s_{R}^{\prime} \geqslant 0$ and hence $\Delta_{k}^{\prime} \geqslant 1+n$ for all $w, k>0$ : assumption $\mathbf{H}$ holds.

Moreover, an interesting sufficient condition for $\mathbf{H}$ is if the utility function has a constant inter-temporal elasticity $\sigma$ and the production function is a CES with an elasticity of substitution $1 /(1+\rho)$. In that case:

$$
\Delta(k, w)=(1+n) k-\frac{w}{1+\beta^{-\sigma} R^{1-\sigma}}
$$

with

$$
R=f^{\prime}(k)=\alpha A^{-\rho}\left(\frac{f(k)}{k}\right)^{1+\rho}
$$

The solution $k$ of $\Delta(k, w)=0$ is obtained by solving

$$
\frac{w}{1+n}=\psi(k) \equiv k+k \beta^{-\sigma} f^{\prime}(k)^{1-\sigma}
$$

for $k$. As $\psi(k)$ is bounded above by $k+k \beta^{-\sigma} f^{\prime}(k) f^{\prime}(1)^{-\sigma}$ for $k<1$ and as $\lim _{k \rightarrow 0} k f^{\prime}(k)=$ 0 , we have that $\psi(0+)=0$. As $\psi(+\infty)=+\infty$, the condition $\psi^{\prime}(k)>0$ for all $k$ guarantees the uniqueness of $k_{t+1}$. Recognizing that $\psi(k)$ can be rewritten as

$$
\psi(k)=k+\beta^{-\sigma}\left(\alpha A^{-\rho}\right) f(k)^{\lambda} k^{1-\lambda} \quad \text { with } \quad \lambda=(1+\rho)(1-\sigma),
$$

the condition $\psi^{\prime}(k)>0$ holds if $\lambda \leqslant 1$, i.e. if

$$
\sigma \leqslant 1-\frac{1}{1+\rho} .
$$

Hence, the condition on $\sigma$ is more restrictive when possibilities for technological substitution are low. When $\rho \leqslant 0$, there is no restriction on $\sigma$.

Under assumption $\mathbf{H}$, the equilibrium is unique and we may apply the implicit function theorem. Equilibrium is then characterized by the sequence of capital stocks $k_{t}$ defined by the difference equation

$$
k_{t+1}=g\left(k_{t}\right) \Longleftrightarrow \Delta\left(k_{t+1}, \omega\left(k_{t}\right)\right)=0
$$


where the derivative of $g$ is

$$
g^{\prime}(k)=\frac{s_{w}^{\prime}\left(\omega(k), f^{\prime}(g(k))\right) \omega^{\prime}(k)}{1+n-s_{R}^{\prime}\left(\omega(k), f^{\prime}(g(k))\right) f^{\prime \prime}(g(k))}>0
$$

and the dynamics are monotonic.

The uniqueness of the equilibrium is a fundamental property to interpret the perfect foresight assumption in a non ad-hoc way. In the case of uniqueness there is no exogenous problem of coordinating expectations, as each agent can solve the model and calculate the next period equilibrium $k_{t+1}$ which is necessary to obtain the rate of return $f^{\prime}\left(k_{t+1}\right)$. In the case of multiplicity of equilibria, agents do not know what the expectations of the others are and thus face a non-unique forward rate of return, unless some exogenous coordination device is assumed (like, e.g., sunspots). Notice finally that a sufficient condition for $\mathbf{H}$ to hold is that savings are non-decreasing in the interest rate.

With the assumption of myopic foresight,

$$
R_{t+1}^{e}=R_{t}=f^{\prime}\left(k_{t}\right)
$$

the inter-temporal equilibrium with initial capital stock $k_{0}$ is necessarily unique and is characterized by the dynamics of $k_{t}$ :

$$
k_{t+1}=\frac{1}{1+n} s\left(\omega\left(k_{t}\right), f^{\prime}\left(k_{t}\right)\right) \equiv m\left(k_{t}\right) .
$$

The derivative of $m(k)$ is

$$
m^{\prime}(k)=\frac{1}{1+n}\left(s_{w}^{\prime}\left(\omega(k), f^{\prime}(k)\right) \omega^{\prime}(k)+s_{R}^{\prime}\left(\omega(k), f^{\prime}(k)\right) f^{\prime \prime}(k)\right) .
$$

\section{The steady states}

Proposition 1 The two dynamics with perfect and myopic foresight have the same positive steady states.

Proof: $\bar{k}>0$ is a steady state for the dynamics with myopic foresight if and only if

$$
\bar{k}=m(\bar{k})=\frac{1}{1+n} s\left(\omega(\bar{k}), f^{\prime}(\bar{k})\right) .
$$

For the dynamics with perfect foresight, $\bar{k}>0$ is a steady state if and only if

$$
\bar{k}=g(\bar{k})=\frac{1}{1+n} s\left(\omega(\bar{k}), f^{\prime}(g(\bar{k}))\right)
$$

and the two conditions are equivalent.

Notice that proposition 1 applies to positive steady states only. For the dynamics described by $x_{t+1}=\phi\left(x_{t}\right)$ with $\phi: \mathbb{R}_{+} \rightarrow \mathbb{R}_{+}$, we say that 0 is a corner steady state if

$$
\phi(0+)=\lim _{x \rightarrow 0+} \phi(x)=0
$$


We can then show by a simple counter-example that when 0 is a corner steady state of the dynamics with myopic foresight it is not necessarily a corner steady state of the dynamics with perfect foresight. The example is as follows: For a CES production function with $\rho<0$ we have $\omega(0+)>0$ and $f^{\prime}(0+)=+\infty$. For a utility function $u^{1-1 / \sigma} /(1-1 / \sigma)$ with $\sigma<1$ we have $s\left(\omega(0+), f^{\prime}(0+)\right)=0$. Hence $m(0+)=0$ but $g(0+)>0$ and 0 is a corner steady state for the dynamics with myopic foresight only. In this situation, although wages are positive at $k=0$, savings are zero in the myopic foresight case because the interest factor forecasted on the basis of the current capital stock is infinite (agents do not forecast that the capital stock can be higher tomorrow). In the perfect foresight case savings are positive as agents expect the interest rate to be finite.

\section{Local stability}

For the myopic dynamics (5), the derivative $m^{\prime}(k)$ is given by (6). The value of this derivative at the steady state determines the local stability of the steady state.

For the rational dynamics, $k_{t+1}=g\left(k_{t}\right)$, we have at a steady state $\bar{k}>0, g(\bar{k})=\bar{k}$ and

$$
g^{\prime}(\bar{k})=\frac{s_{w}^{\prime}\left(\omega(\bar{k}), f^{\prime}(\bar{k})\right) \omega^{\prime}(\bar{k})}{1+n-s_{R}^{\prime}\left(\omega(\bar{k}), f^{\prime}(\bar{k})\right) f^{\prime \prime}(\bar{k})}>0 .
$$

If $g^{\prime}(\bar{k})<1, \bar{k}$ is locally stable. Under assumption $\mathbf{H}$ this is equivalent to $s_{w}^{\prime} \omega^{\prime}<$ $1+n-s_{R}^{\prime} f^{\prime \prime}$ or to $m^{\prime}(\bar{k})<1$ (but $m^{\prime}(\bar{k})$ may be smaller than -1 ). If $g^{\prime}(\bar{k})>1, \bar{k}$ is unstable. The condition $g^{\prime}(\bar{k})>1$ is equivalent to $m^{\prime}(\bar{k})>1$.

Proposition 2 Consider a steady state $\bar{k}>0$ and assume $\mathbf{H}$.

In the case where $m^{\prime}(\bar{k}) \geqslant 0$ (monotonic dynamics with myopic foresight), $\bar{k}$ is respectively stable, unstable or non-hyperbolic for the two dynamics when $m^{\prime}(\bar{k})$ is respectively $<1$, $>1$ or $=1$.

In the case where $m^{\prime}(\bar{k})<0, \bar{k}$ is stable for the rational dynamics but may be stable $\left(m^{\prime}(\bar{k})>-1\right)$ or unstable $\left(m^{\prime}(\bar{k})<-1\right)$ for the myopic dynamics.

Proof: When $m^{\prime}(\bar{k}) \geqslant 0$ we have $\left|m^{\prime}(\bar{k})\right|=m^{\prime}(\bar{k})$ and the first order stability conditions for the two dynamics are identical. When $m^{\prime}(\bar{k})<0, s_{w}^{\prime} \omega^{\prime}<1+n-s_{R}^{\prime} f^{\prime \prime}, g^{\prime}(\bar{k})<1$ and $\bar{k}$ is stable for the rational dynamics.

Corollary: When the rational dynamics are defined (hyp. $\mathbf{H})$ and the myopic dynamics are monotonic, the two dynamics have the same structure in the sense that the positive steady states are the same and the first order stability conditions are equivalent.

This is a useful result in the case of monotonic myopic dynamics: indeed, the study of these dynamics, which are much simpler, allows to characterize the dynamics under perfect foresight.

\section{Uniqueness of the steady state}

The savings function depends on the first derivatives of $u$ and $f$. A general condition which is sufficient for uniqueness, e.g. the concavity of $g$, involves the third derivatives of 
$u$ and $f$ (see Galor and Ryder (1989)).

However, to study the number of steady states, we can use the results on the myopic dynamics and derive a simpler sufficient condition for uniqueness.

Proposition 3 No more than one positive steady state $\bar{k}$ of the dynamics with perfect foresight exists when

$$
s_{w}^{\prime}\left(\omega(k), f^{\prime}(k)\right) \omega^{\prime}(k)+s_{R}^{\prime}\left(\omega(k), f^{\prime}(k)\right) f^{\prime \prime}(k)<\frac{s\left(\omega(k), f^{\prime}(k)\right)}{k}, \quad \forall k>0
$$

Such a steady state exists if and only if

$$
\lim _{k \rightarrow 0} \frac{s\left(\omega(k), f^{\prime}(k)\right.}{k}>1+n
$$

Proof: No more than one positive steady state $\bar{k}$ exists if the function $s\left(w(k), f^{\prime}(k)\right) / k$ is strictly decreasing, i.e.

$$
\frac{\mathrm{d}}{\mathrm{d} k} \frac{\left.s\left(w(k), f^{\prime}(k)\right)\right)}{k}=\frac{1}{k}\left(s_{w}^{\prime} \omega^{\prime}+s_{R}^{\prime} f^{\prime \prime}-\frac{s}{k}\right)<0, \quad \forall k>0 .
$$

This condition can be rewritten

$$
m^{\prime}(k)<\frac{1}{1+n} \frac{s\left(\omega(k), f^{\prime}(k)\right)}{k}, \forall k>0
$$

which leads to the expression in the proposition.

The function $s\left(w(k), f^{\prime}(k)\right) / k$ starts above $1+n$ if and only if $\lim _{k \rightarrow 0} \frac{s\left(w(k), f^{\prime}(k)\right.}{k}>1+n$ holds, and, as it is decreasing and goes to 0 , takes the value $1+n$ only once?. Hence the steady state is unique.

As $s\left(\omega(k), f^{\prime}(k)\right)<\omega(k)$, a necessary condition of existence is $\lim _{k \rightarrow 0} \omega(k) / k>1+n$ (see Galor and Ryder (1989)).

The interest of this proposition is to propose a condition of uniqueness that bears only on the second derivatives of the functions $u$ and $f$.

\section{Example}

To illustrate the conclusions of the this note, we take a CES production function $f(k)=$ $\left.A\left(\alpha k^{-\rho}+1-\alpha\right)\right)^{-1 / \rho}$ and the following utility function:

$$
\frac{c^{1-1 / \sigma}+\beta d^{1-1 / \sigma}}{1-1 / \sigma}
$$

We assume that $A=10, n=1.01^{30}-1, \beta=0.98^{30}$ and $\alpha=0.35$. In the first panel of figure 1 we have chosen a production function with high substitution possibilities, $\rho=-0.5$, and a low level of the inter-temporal elasticity of substitution, $\sigma=.5$. The dotted line represents the function $k_{t+1}=g\left(k_{t}\right)$ (perfect foresight) and the solid line represents the function $k_{t+1}=m\left(k_{t}\right)$ (myopic foresight). Figure 1 illustrates the fact that the corner 

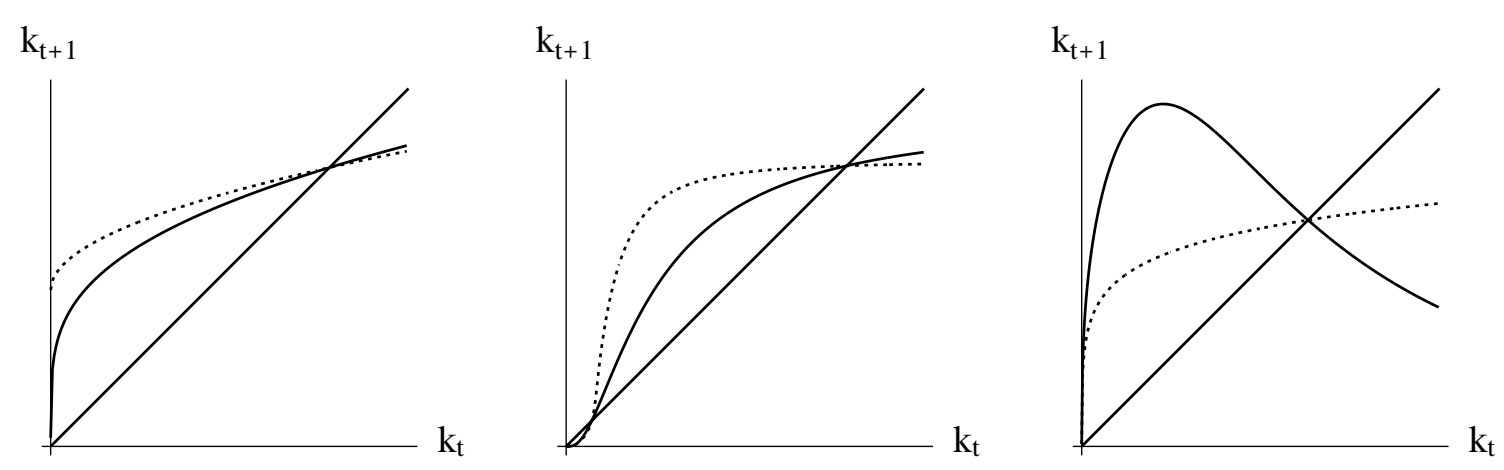

Figure 1: Examples

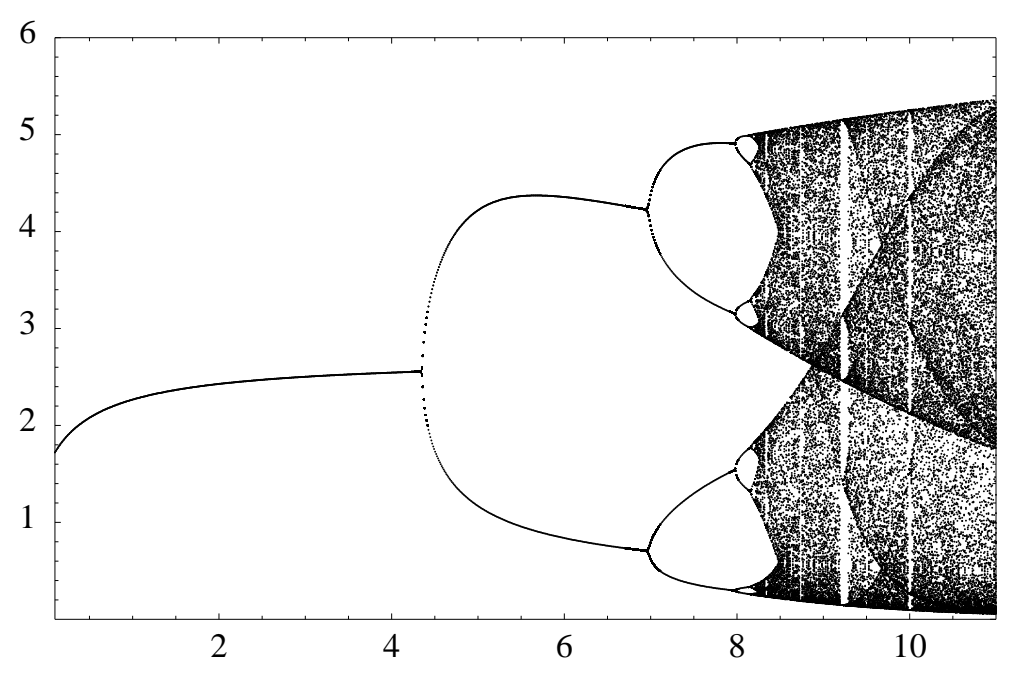

Figure 2: Bifurcation diagram for $\sigma$

steady state can be different in the two dynamics. In the second panel of figure 1 , we keep $\sigma=.5$ and take a production function with low substitution possibilities, $\rho=2$. As the rational dynamics are defined and the myopic dynamics are monotonic, studying the myopic dynamics is sufficient to characterize the stability type of the steady states under perfect foresight. In the last panel we have chosen a Cobb-Douglas production function, $\rho \rightarrow 0$, and a high value of the inter-temporal elasticity of substitution, $\sigma=4$. In this case, the dynamics with myopic foresight are oscillatory. For these parameter values, the positive steady state is stable in both cases.

We have seen in the last panel of Figure 1 that the dynamics under myopic foresight oscillate near $k^{\star} ; k^{\star}$ is stable as long as $m^{\prime}\left(k^{\star}\right)>-1$. When $m^{\prime}\left(k^{\star}\right)<-1, k^{\star}$ is unstable for the myopic dynamics. In this case the two steady states $k^{\star}$ and 0 are unstable but the dynamics are bounded, as the function $m$ has a finite maximum. To analyze the effect of the inter-temporal elasticity of substitution on the nature of the dynamics we present the bifurcation diagram (figure 2) for the parameter $\sigma$ given the other parameters $(\rho=0) . \sigma$ lies on the horizontal axis, and we plot vertically limit values of the equilibrium sequences $\left(k_{t}\right)$. For relatively low $\sigma$ we observe a unique limit point which is a stable steady state. There is then an interval in which period doubling bifurcations occur more and more 
rapidly, showing the existence of deterministic stable cycles. Beyond a certain value, one enters into the chaotic region.

\section{Conclusion}

In this paper we have compared the dynamics under myopic foresight and those under perfect foresight in an overlapping generations model with capital accumulation. Our results are the following

- The two dynamics have the same positive steady state(s). When 0 is a "corner" steady state of the dynamics with myopic foresight, it is not necessary a "corner steady state" of the dynamics with perfect foresight.

- When the rational dynamics are defined and the myopic dynamics are monotonic, the study of myopic dynamics, which are much simpler, allows to characterize the dynamics under perfect foresight.

- The study of myopic dynamics allows to obtain a condition of uniqueness of the steady state that bears only on the second derivatives of the functions $u$ and $f$.

\section{References}

Diamond, P. (1965) "National debt in a neoclassical growth model". American Economic Review, 55:1126-1150.

Galor, O. and H. Ryder (1989) "Existence, uniqueness and stability of equilibrium in overlapping generations model with productive capital". Journal of Economic Theory, 49:360-375. 